\title{
Saúde urbana, ambiente e desigualdades
}

\author{
Urban health, environment, and inequalities \\ Salud urbana, ambiente y desigualdades \\ Maria Inês Reinert Azambuja ${ }^{1 *}$, Aloyzio Cechella Achutti², Roberta Alvarenga Reis ${ }^{3}$, Jacqueline Oliveira Siva ${ }^{4}$, \\ Paul Douglas Fisher ${ }^{5}$, Roger dos Santos Rosa ${ }^{6}$, Ronaldo Bordin ${ }^{7}$, Francisco Arsego de Oliveira ${ }^{8}$, \\ Roger Keller Celeste ${ }^{9}$, Aline Petter Schneider ${ }^{10}$, Darci Barnech Campani ${ }^{11}$, Lívia Piccinini ${ }^{12}$, \\ Maurem Ramos ${ }^{13}$, Miguel Aloysio Sattler ${ }^{14}$, Paulo Antonio Barros Oliveira ${ }^{15}$, Alzira Maria Baptista Lewgoy ${ }^{16}$
}

Palavras-chave: Saúde da População Urbana Meio Ambiente Desigualdades em Saúde

Keywords: Urban Health Environment Health Inequalities

\section{Resumo}

Os ambientes psicossocial, econômico e físico, nos quais se nasce, cresce, vive e trabalha, afetam a saúde e a longevidade, tanto quanto 0 fumo, o exercício e a dieta. A atenção individual à saúde não é suficiente para prevenir ou controlar os efeitos das más condições ambientais. Evidências históricas e atuais apontam para o agravamento das condições de saúde das populações mais pobres, acompanhando processos de urbanização rápida. Esperadamente, 0 envelhecimento populacional num ambiente urbano de desigualdade social deverá agravar a situação de saúde da população mais pobre, resultando em mais sofrimento e em perdas econômicas para o país. Com base nestas justificativas, um grupo de professores da Universidade Federal do Rio Grande do Sul se organizou, via extensão universitária, para contribuir com a discussão e as iniciativas nacionais de intervenção sobre a saúde urbana. Os projetos do grupo abarcam: o debate sobre o impacto potencial de iniciativas privadas e políticas públicas setoriais (de habitação, saneamento, transporte, educação, inovação tecnológica, sustentabilidade ambiental etc.) na saúde urbana; a produção e divulgação de conteúdos sobre determinantes sociais e ambientais da saúde; a produção e disseminação dos indicadores de desigualdade social dos determinantes da saúde; a formação de recursos humanos; e a participação em redes sociais. A apresentação pública deste projeto cumpre o objetivo de contribuir desde já com essa discussão.

\section{Abstract}

The psychosocial, economic, and physical environment where we were born, and now are growing, living, working, and reproducing affect health and longevity as much as do smoking, exercise, and diet. Individual attention to health is not enough to prevent or control the effects of bad environmental conditions. Historical and current evidence show that a decline in the health condition of the poorest is associated with rapid urbanization. Population aging in an urban environment of social inequalities is expected to make the health situation of the poorest even worse, resulting in more human suffering and economic losses for the country. Based on these observations, a group of professors from

\footnotetext{
Universidade Federal do Rio Grande do Sul (UFRGS). Porto Alegre (RS), Brasil. miazambuja@terra.com.br.

${ }^{2}$ Universidade Federal do Rio Grande do Sul (UFRGS). Porto Alegre (RS), Brasil. achutti@gmail.com.

${ }^{3}$ Universidade Federal do Rio Grande do Sul (UFRGS). Porto Alegre (RS), Brasil. robfono@gmail.com.

${ }^{4}$ Universidade Federal do Rio Grande do Sul (UFRGS). Porto Alegre (RS), Brasil. jacquesilva@click21.com.br.

${ }^{5}$ Universidade Federal do Rio Grande do Sul (UFRGS). Porto Alegre (RS), Brasil. paul.fisher@ufrgs.br.

${ }^{6}$ Universidade Federal do Rio Grande do Sul (UFRGS). Porto Alegre (RS), Brasil. roger.rosa@bcb.gov.br.

${ }^{7}$ Universidade Federal do Rio Grande do Sul (UFRGS). Porto Alegre (RS), Brasil. Ronaldo.Bordin@ufrgs.br.

${ }^{8}$ Universidade Federal do Rio Grande do Sul (UFRGS). Porto Alegre (RS), Brasil. arsego@via-rs.net.

${ }^{9}$ Universidade Federal do Rio Grande do Sul (UFRGS). Porto Alegre (RS), Brasil. roger.keller@ufrgs.br.

10Universidade Federal do Rio Grande do Sul (UFRGS). Porto Alegre (RS), Brasil. aline@ipgs.com.br.

${ }^{11}$ Universidade Federal do Rio Grande do Sul (UFRGS). Porto Alegre (RS), Brasil. campani@ufrgs.br.

${ }^{12}$ Universidade Federal do Rio Grande do Sul (UFRGS). Porto Alegre (RS), Brasil. liviapiccinini@hotmail.com.

${ }^{13}$ Universidade Federal do Rio Grande do Sul (UFRGS). Porto Alegre (RS), Brasil. maurem.profnut@gmail.com.

${ }^{14}$ Universidade Federal do Rio Grande do Sul (UFRGS). Porto Alegre (RS), Brasil. masattler@gmail.com.

${ }^{15}$ Universidade Federal do Rio Grande do Sul (UFRGS). Porto Alegre (RS), Brasil. oliveira.pauloantonio@gmail.com.

${ }^{16}$ Universidade Federal do Rio Grande do Sul (UFRGS). Porto Alegre (RS), Brasil. lewgoy@terra.com.br.

${ }^{*}$ Autor correspondente

Fonte de financiamento: MEC - PROEXT

Conflito de interesse: declararam não haver

Recebido em: 07/03/2011

Aprovado em: 12/04/2011
} 
the Universidade Federal do Rio Grande do Sul (UFRGS) organized themselves, through an extension program, to contribute on the discussion and national initiatives of intervention in urban health. The group's projects are comprised of: discussions about the potential impact of private initiatives and sector public policies (housing, sanitation, transportation, education, technological innovation, environmental sustainability, and so on) on urban health; the production and dissemination of information about social and environmental determinants of health; the production and dissemination of new indicators of inequality as determinants of health; human resources development; and the participation in social networks. The public presentation of this project satisfies the objective of initiating the group's contribution to this discussion.

Palabras clave:

Salud Urbana Ambiente

Desigualdades en la Salud

\section{Resumen}

Los ambientes psicosocial, económico y físico en los cuales nacemos, crecemos, vivimos, trabajamos y nos reproducimos afectan la salud y la longevidad, tanto como el tabaco, el ejercicio y la dieta. La atención individual a la salud no es suficiente para prevenir 0 controlar los efectos de malas condiciones ambientales. Evidencias históricas y actuales muestran el agravamiento de las condiciones de salud de las poblaciones más pobres acompañando procesos de urbanización rápida. Previsiblemente, el envejecimiento de la población en un ambiente urbano de desigualdad social deberá agravar la situación de la salud de la población más pobre, resultando en más sufrimiento y en pérdidas económicas para el país. Con base en estas justificativas, un grupo de profesores de la Universidade Federal do Rio Grande do Sul (UFRGS) se organizó, a través de la extensión universitaria, para contribuir con la discusión y las iniciativas nacionales de intervención sobre la Salud Urbana. Los proyectos del grupo abarcan: el debate sobre el impacto potencial de iniciativas privadas y políticas públicas sectoriales (de habitación, saneamiento, transporte, educación, innovación tecnológica, sustentabilidad ambiental, etc.) en la Salud Urbana; la producción y divulgación de contenidos sobre determinantes sociales y ambientales de la salud; la producción y diseminación de indicadores de desigualdad social como determinantes de la salud; la formación de recursos humanos; y la participación en redes sociales. La presentación pública de este proyecto cumple con el objetivo de contribuir desde ya con esta discusión.

\section{Introdução}

BRUNNER: Os maiores problemas de saúde são extremamente complicados e profundos, e requerem um amplo espectro de estratégias, da educação dos indivíduos, mobilização da comunidade e da vizinhança, à construção de parcerias e mudanças nas políticas públicas.

BLACKWELL: A primeira coisa necessária é reconbecer que onde você vive impacta na sua saúde. Que o ambiente na comunidade, o ambiente social, o físico e o econômico juntos determinam se teremos ou não uma existência saudável.

WILLIAMS: Isto significa que política de habitação épolítica de saúde. Educação é política de saúde. Política antiviolência é politica de saúde. Políticas de melhorias nos bairros são politicas de saúde. Tudo que nós fizermos para melhorar a qualidade de vida dos indivíduos na sociedade tem um impacto na sua saúde e épolítica de saúde.

Esse é o diálogo final do vídeo "Unnatural causes: place matters" , da série "Unnatural causes: ... is inequalities making us sick?" 2 , lançada pela PBS, em 2008. A série discute as evidências de que os ambientes psicossocial, econômico e físico nos quais se nasce, cresce, vive e trabalha afetam a nossa saúde e longevidade, tanto quanto o fumo, o exercício e a dieta.

Usualmente, quando se fala em saúde, pensa-se na doença, em seus mecanismos biológicos, em fatores de risco e no acesso ao tratamento. Mas, saúde é harmonia, equilíbrio, bem-estar ${ }^{3}$. Manter-se saudável depende da interação entre o ambiente e a capacidade de adaptação a $\operatorname{ele}^{3-4}$ ou resiliência ${ }^{4}$. Mesmo com ênfases em aspectos diferentes, cada vez mais têm sido discutidos os efeitos deletérios do estresse decorrente das relações sociais na capacidade de adaptação dos indivíduos ao ambiente ${ }^{5-11}$. Enquanto uns autores enfocam as relações sociais entre indivíduos e entre grupos na vizinhança, outros enfatizam os contextos sociais, políticos e econômicos que as antecederam e condicionam $^{7}$ e que persistem cristalizados na cultura e nos lugares $^{12}$ (a habitação, o bairro, os ambientes de trabalho e a distribuição espacial dos equipamentos urbanos), tendendo a reproduzir os processos que lhes deram origem. Ambiente, relações sociais e saúde são, portanto, indissociáveis; e promover a saúde, nesta perspectiva, requer ir além dos serviços de saúde.

\section{Justificativa}

Há coincidências significativas entre a situação atual das grandes cidades brasileiras e as cidades europeias do início do século 19 $13^{13-17}$. Tanto na Europa como no Brasil, a industrialização promoveu um rápido aumento da população urbana, com "favelização" e grande desigualdade social na mortalidade ${ }^{13,15-17}$. A população de Paris e de Londres aumentou cinco vezes, e a de Berlim, dez vezes nos 100 anos da revolução industrial ${ }^{15}$. Já a população brasileira habitando cidades passou de 19 para 146 milhões de habitantes entre 1950 e $2000^{18}$, ou seja, aumentou mais de sete vezes em apenas 50 anos.

Entre 1830 e 1840, Villermé, na França, e Chadwick, na Inglaterra, mostraram que a mortalidade era maior nas grandes cidades. Villermé mostrou que a mortalidade era 
$50 \%$ maior nos distritos mais pobres ${ }^{15}$. A tuberculose, seguida pela pneumonia e pela influenza eram, então, as principais causas de morte nas cidades europeias ${ }^{15}$.

Em Londres, as precárias condições de vida reconhecidamente favoreciam grande mortalidade nas epidemias, especialmente nas de cólera, que acometeram a cidade em 1833 e em 1848 e $1849^{19}$. A má saúde da população ganhava dimensões de questão de Estado. Era discutido se a pobreza causava a doença ou a doença causava a pobreza ${ }^{13-17}$, mas doença e pobreza eram reconhecidas como entraves ao desenvolvimento da Inglaterra ${ }^{16}$.

Em 1848, Chadwick - o pai da saúde pública inglesa - defendeu e conseguiu aprovar o Public Health Act (Lei da Saúde Pública). O argumento de Chadwick era que investimentos em obras de engenharia sanitária, visando melhoria das condições de habitação e saneamento da cidade, além de beneficiarem diretamente a saúde, favoreceriam o emprego e a renda e, assim, a melhoria nas condições de vida na cidade ${ }^{16}$. Mesmo sob a ameaça da cólera, a aprovação do Public Health Act não foi tranquila ${ }^{17}$, e custou à Chadwick seu cargo de 30 anos como funcionário público exemplar ${ }^{16}$.

A situação da Inglaterra de 1848 não difere muito da realidade dos grandes centros urbanos dos países menos desenvolvidos atualmente ${ }^{9}$, entre eles, o Brasil. A tuberculose foi substituída pelas doenças do aparelho circulatório como primeira causa de óbito nas populações, mas para esta e outras causas de adoecimento e morte, persistem desigualdades sociais significativas na distribuição dos $\operatorname{casos}^{20-24}$.

Em Porto Alegre, capital estadual que, em 2000, tinha o segundo melhor índice de desenvolvimento humano do Brasil, a mortalidade precoce por doenças do coração no período de 2000 a 2004 chegava a ser três vezes maior no bairro com os piores indicadores sociais, comparado ao com os melhores indicadores ${ }^{20}$. O mesmo ocorria com a mortalidade por doenças respiratórias ${ }^{23}$. Para a tuberculose, o coeficiente era 7,9 maior $^{24}$.

Ainda em Porto Alegre, o indicador que resume o risco cumulativo de morrer por qualquer causa como seu inverso - a esperança de vida ao nascer - apontava diferenças de dez anos entre os homens residentes em bairros nos extremos da distribuição da qualidade de vida, ou seja, mantidas as diferenças nas taxas de mortalidade por idade calculadas para o período entre 2000 a 2004, os homens nascidos no bairro mais pobre atualmente poderiam esperar viver em média dez anos menos do que aqueles nascidos no mais rico ${ }^{25}$.

Vivem-se os anos que deveriam proporcionar ao país o que os demógrafos chamam de "bônus demográfico", ou seja, uma vantagem econômica decorrente da estrutura etária da população ${ }^{26}$. Este bônus ocorreria quando a proporção de adultos em idade produtiva superaria significativamente à de dependentes econômicos, nos dois extremos da vida. No caso do Brasil, os benefícios máximos ocorreriam entre 1970, quando se intensificou a redução da natalidade, e 2020, quando a proporção de idosos aposentados torna-se significativa com relação à de trabalhadores ativos ${ }^{26}$.

Mas o bônus demográfico pressupõe um ambiente econômico adequado, e adultos saudáveis com condições de empregabilidade. Sabe-se que essas não foram as condições dos últimos 40 anos, ao contrário. Assim, pode-se ter perdido parcialmente esta oportunidade, e provavelmente avança-se para a próxima fase, de maior proporção de idosos, levando junto uma parcela significativa de adultos não-produtivos, por doença precoce ou por falta de investimento passado em sua qualificação $0^{27-29}$. As cidades, que hoje concentram aproximadamente $80 \%$ da população brasileira, deverão suportar o maior peso desta crise anunciada.

Como identificado por Chadwick, a relação entre pobreza e doença tem duas mãos. Como resolver essa equação? Com mais serviços de saúde para a redução da doença? Com benefícios sociais para a redução da pobreza? Com investimentos pensados para reduzir ao mesmo tempo a pobreza e a doença, como fez Chadwick ao investir em saneamento? Com investimentos nas relações sociais, possivelmente as causa das causas das causas?

Como somar à produção e disseminação de conhecimentos já em curso ${ }^{4,6-10,30-33}$, e contribuir para a redução das iniquidades e a melhoria da saúde urbana?

\section{Marco referencial para a ação: investir em produtos ou processos?}

Cada vez mais, em todos os campos do saber, o interesse dos pesquisadores tem-se voltado para os processos organizativos. A economia ${ }^{34}$ e o direito ${ }^{35-36}$ têm avançado na exploração deste paradigma chamado "da complexidade", assim como a biologia ${ }^{37}$ e a própria saúde pública ${ }^{38}$. Em artigo de 2011, Jayasighe faz a defesa da aplicação da ciência da complexidade à saúde populacional, como alternativa à visão mais mecanicista da realidade que evoluiu a partir da mecânica Newtoniana ${ }^{39}$. Em um artigo recente ${ }^{37}$, Kurakin sumariza muito bem conceitos associados à complexidade e, segundo o próprio autor, aplicáveis a todas as organizações vivas, de proteínas e células a nichos ecológicos e sociedades.

O primeiro conceito é que, nos seres vivos, os constituintes funcionais, ou seja, os processos que mantêm as es- 
truturas vivas, são eles mesmos também organizações vivas, com "estruturas" e dinâmicas formatadas, mas não determinadas, pela evolução.

O segundo é que as estruturas e os processos das organizações vivas têm nelas incorporada a história/memória, a qual ao mesmo tempo facilita e parcialmente governa (mas não determina) a sua contínua auto-organização. A memória das experiências anteriores está acumulada em diferentes níveis, e se reproduz como estruturas e processos dos subsistemas, e é isto que torna a reprodução do sistema todo mais rápida e eficiente, à medida que as memórias se acumulam. Como disse Nelson Rodrigues, "O subdesenvolvimento não se improvisa. É fruto de séculos"

Conforme Kurakin ${ }^{37}$, outras leis da auto-organização de sistemas termodinâmicos fora do equilíbrio, que são úteis para entender a dinâmica organizacional dos sistemas vivos, inclusive sociais, seriam: aumentar o fluxo de energia/matéria por meio de um sistema de componentes interativos normalmente levaria ao crescimento na organização do sistema em tamanho e complexidade, em geral, com o desenvolvimento de formas hierárquicas (multinível) de organização; manter um sistema de nãoequilíbrio num mesmo nível de organização requer um fluxo constante de energia/matéria por meio do sistema; reduzir o fluxo de energia/matéria leva a um relaxamento da estrutura organizacional e à perda da complexidade e da ordem, culminando com a dissolução e morte da organização.

Segue-se destas leis empíricas que os parâmetros críticos para definir o estado de qualquer sistema vivo são os fluxos de seus constituintes e não as concentrações. Milton Santos afirma que não é a produção que comanda, mas a circulação. "Não adianta pedir às pessoas que produzam se elas não puderem também organizar a circulação. A redistribuição se dá pela via da circulação" ${ }^{1}$.

Diferentes configurações de fluxos de energia/matéria e suas manifestações metaestáveis continuamente competem e cooperam entre si de forma a se manterem ou crescerem em tamanho ou complexidade. Analogamente, fluxos de recursos articulados sob diferentes configurações, envolvendo negócios e organizações sociais e políticas, competem e cooperam entre si de modo a crescerem dentro do sistema sociopolítico econômico que eles constituem. Aquelas configurações que conseguem assegurar e acelerar os fluxos de recursos/energia por meio de suas estruturas crescem em tamanho, ordem, complexidade e influência. Aquelas que falham, diminuem seu tamanho relativo, complexidade e influência ou se dissolvem ${ }^{37}$.

Milton Santos fala nas "variáveis dinâmicas que dão conta do caminho para o futuro”¹1. Na área da saúde pública,
Almeida-Filho e Coutinho falam da emergência (do que ainda não é do futuro) como resultado de processos múltiplos estruturados em redes, sem relação linear de causalidade, mas que no lugar, no contexto, entendidos como integrando uma situação contingente, poderiam orientar propostas para a promoção da saúde ${ }^{38}$.

Seria possível intervir no processo social por meio do favorecimento/aceleração de alguns fluxos em competição com outros?

\section{Proposta de ação}

O Ministério da Saúde tem enfatizado a necessidade dos profissionais de saúde terem um conhecimento aprofundado do território de atuação de seus serviços como elemento fundamental para o desenvolvimento de um processo de trabalho efetivo na atenção primária ${ }^{42}$. Mas, para além da assistência, como ampliar a atuação dos médicos na promoção da saúde?

$\mathrm{Na}$ universidade, os recursos à disposição são a informação e a produção e circulação de conhecimentos. Uma possibilidade então seria aumentar a quantidade de conhecimento/informação sobre os efeitos de investimentos setoriais (em habitação, saneamento, transporte, educação, inovação tecnológica, sustentabilidade ambiental etc.) sobre a saúde urbana. Outra seria investir em mais conhecimento e informações sobre os efeitos das relações interpessoais e intergrupais nas desigualdades sociais e na saúde, no meio urbano. Aumentos dos fluxos de informação nestas e em outras áreas sensíveis talvez, numa "disputa evolutiva", favoreceriam um ambiente e processos propícios à redução das iniquidades em saúde.

Com estes desafios em mente e o estímulo do grupo de Saúde Ambiental da OPAS/Washington (Teixeira P, Galvão LA), um grupo de professores do Departamento de Medicina Social da Universidade Federal do Rio Grande do Sul (UFRGS) vem trabalhando, desde janeiro de 2010, na ampliação da discussão sobre saúde para além do horizonte dos serviços de saúde e da atenção à saúde individual. $\mathrm{O}$ grupo se organizou inicialmente em torno de um Programa de Extensão e Pesquisa em Saúde Urbana, Ambiente e Desigualdades, com o objetivo construir parcerias internamente na UFRGS, e com outras instituições públicas e privadas, locais e globais, interessadas no desenvolvimento urbano sustentável e equitativo, requisito para a saúde da população. Foi promovido, na Universidade, um seminário interdisciplinar sobre saúde urbana ${ }^{43}$ no Dia Mundial da Saúde (sete de abril), com boa audiência. Já com a participação de professores de outras unidades (Engenharia, Ar- 
quitetura, Odontologia, Fonoaudiologia, Nutrição) e parceria do Centro Estadual de Vigilância em Saúde (CEVS) da Secretaria Estadual da Saúde (Paz F, Sperb AF, Fernandes M), o grupo participou com sucesso de editais do MEC do Programa PET-Vigilância em Saúde e PROEXT 2010, e está agora se organizando como núcleo de extensão e pesquisa multidisciplinar em Saúde Urbana, com projetos que devem abarcar diferentes territórios da área de cobertura de uma Unidade de Saúde da Família e da Comunidade à cidade e à área metropolitana. Os instrumentos à disposição do grupo são seminários favorecendo o debate intersetorial sobre o impacto potencial de iniciativas privadas e políticas públicas (de habitação, saneamento, transporte, educação, inovação tecnológica, sustentabilidade ambiental etc.), na saúde urbana; a produção e divulgação de conteúdos sobre determinantes sociais e ambientais da saúde; a produção e disseminação de indicadores de desigualdade social dos determinantes da saúde; a formação de recursos humanos; e a participação em redes sociais.

A apresentação deste programa de extensão ainda em fase inicial de desenvolvimento visa convidar a todos, desde já, a intensificar o debate público sobre a saúde urbana, o meio ambiente e as desigualdades no Brasil.

\section{Referências}

1. Unnatural causes - places matter... is inequality making us sick? California Newsreel Production 2008. Public Engagement Campaign in Association with the Joint Center for Political and Economic Studies Health Policy Institute. [acesso em 2010 Abr 9]. Disponível em: http://www.unnaturalcauses.org/assets/uploads/file/UC_Transcript_5.pdf

2. Unnatural causes... is inequality making us sick? California Newsreel Production 2008. Public Engagement Campaign in Association with the Joint Center for Political and Economic Studies Health Policy Institute. [acesso em 2010 Abr 9]. Disponível em: http://www.pbs.org/unnaturalcauses/about the series.htm.

3. Achutti A. A partir do coração. Jornal Zero Hora. 2010; 16417

4. Friedli L. Mental health, resilience and inequalities. World Health Organization, Regional Office for Europe, 2009. [acesso em 2010 Aug 03]. Disponível em: http://www.euro.who.int/_data/assets/pdf_file/0012/100821/E92227.pdf.

5. McEwen BS. Stress, adaptation, and disease: Allostasis and allostatic load. Ann NY Acad Sci. 1998; 840: 33-44.

6. Marmot M. The status syndrome: how social standing affects our health and longevity. London: Henry Holt and Co.; 2005.

7. Wilkinson R. Unhealthy societies. New York: Routledge; 1997

8. Macinko J, Starfield B. The Utility of Social Capital in Research on Health Determinants. Milb Quart. 2001; 79: 387-427.

9. Krieger N, Alegría M, Almeida-Filho N, Barbosa da Silva J, Barreto ML, Beckfield J et al. Who, and what, causes health inequities? Reflections on emerging debates from an exploratory Latin American/North American workshop. J Epidemiol Community Health. 2010; 64(9): 747-9.

10. Levins R, Lopez C. Towards an ecosocial view of health. Int J Health Serv. 1999; 29: 261-93.

11. Slavicha GM, Way BM, Eisenberger NI, Taylor SE. Neural sensitivity to social rejection is associated with inflammatory responses to social stress. Proc Natl Acad Sci USA. 2010; 107(33): 14817-22.

12. Johnson S. Emergência. A vida integrada de formigas, cérebros, cidades e softwares. Rio de Janeiro: Jorge Zahar; 2003.
13. Szreter $\mathrm{S}$. The population health approach in historical perspective. Am J Public Health. 2003; 93: 421-31.

14. Szreter S. Industrialization and health. Br Med Bull. 2004; 69: 75-86.

15. Cairnes J. Matters of life and death: perspectives on public health, molecular biology, cancer and the prospects for the human race. New Jersey: Princeton University Press; 1997.

16. Susser E, Bresnahan M. Origins of epidemiology. Ann NY Acad Sci. 2001: 954: 6-18

17. Hemenway D. Why we don't spend enough on Public Health. New Engl J Med. 2010;362;18. [acesso em 2010 Apr 9]. Disponível em: http://healthcarereform.nejm.org/?p=3400\&query=TOC

18. Rocha RM. A ocupação e o processo de urbanização sem planejamento no eixo rodoviário do complexo territorial Brasília-Goiânia. Brasília: Faculdade de Arquitetura e Urbanismo - Programa de Pós Graduação; 2006. p. 3.

19. Snow SJ. Commentary: Sutherland, Snow and water: the transmission of cholera in the nineteenth century. Int J Epidemiol. 2002; 31(5): 908-11.

20. Bassanesi SL, Azambuja MI, Achutti AC. Mortalidade precoce por doenças cardiovasculares e desigualdades sociais em Porto Alegre: da evidência à ação. Arq Bras Cardiol. 2008; 90: 403-12.

21. de Souza MF, Alencar AP, Malta DC, Moura L, Mansur AP. Serial tempora analysis of ischemic heart disease and stroke death risk in five regions of Brazil from 1981 to 2001. Arq Bras Cardiol. 2006; 87: 735-40.

22. Ishitani LH, Franco GC, Perpétuo IH, França E. Desigualdade socia e mortalidade precoce por doenças cardiovasculares no Brasil. Rev Saúde Pública. 2006; 40: 684-91.

23. Azambuja MIR, Bassanesi SL, Achutti AC. A mortalidade por doenças respiratórias em Porto Alegre é maior em áreas da cidade com piores indicadores sociais. Bol Saúde. 2009; 23: 31-9.

24. Acosta LMW. O mapa de Porto Alegre e a tuberculose: distribuição espacial e determinantes sociais. Dissertação de Mestrado. PPG Epidemiologia - UFRGS; 2008

25. Bassanesi SL, Azambuja MI, Achutti AC. Life-expectancy and Socia Inequalities across Porto Alegre city districts. In: XVIII IEA World Congress of Epidemiology and VII Brazilian Congress of Epidemiology, 2008, Porto Alegre. Annals XVIII IEA World Congress of Epidemiology; 2008.

26. Alves JED, Vasconcelos DS, Carvalho AA. Estrutura etária, bônus demográfico e população economicamente ativa no Brasil: cenários de longo prazo e suas implicações para o mercado de trabalho. Texto para Discussão Cepal Ipea. 2010; 10: 1-36.

27. Leeder S, Raymond S, Greenberg H, Liu H. A race against time: the challenge of cardiovascular disease in developing economies. The Earth Institute: Columbia University; New York; 2004. Disponível em: http://www.earth.columbia.edu/news/2004/images/raceagainsttime_ FINAL 0410404.pdf.

28. Azambuja MI, Foppa M, Maranhão MFC, Achutti AC. Economic burden of severe cardiovascular diseases in Brazil: an estimate based on secondary data. Arq Bras Cardiol. 2008; 91: 148-55.

29. Achutti AC, Azambuja Ml. Doenças crônicas não-transmissíveis no Brasil: repercussões do modelo de atenção à saúde sobre a seguridade social. Cienc Saúde Col. 2004; 9: 833-40.

30. Víctora C, Barros F. Infant mortality due to perinatal causes in Brazil: trends, regional patterns and possible interventions. São Paulo Med J. 2001; 119: 33-42.

31. Buss PM, Pellegrini Filho A. A saúde e seus determinantes sociais. Physis. 2007;(1). [acesso em 2010 Aug 4]. Disponível em: http://www.scielo.br/scielo.php?script=sci_arttext\&pid=S0103$73312007000100006 \&$ lng=en\&nrm=iso.

32. Comissão Nacional dos Determinantes Sociais da Saúde (CNDSS). Carta aberta aos candidatos à Presidência da República. Setembro de 2006. [acesso em 2010 Aug 03]. Disponível em: www. determinantes. fiocruz.br.

33. Fleury S. "Saúde Urbana e Cidadania", Divulgação em Saúde para Debate: Revista do Centro Brasileiro de Estudos de Saúde. CEBES. 2007;40:29-40.

34. W. Brian Arthur. Complexity and the Economy. Science. 1999;284:107-9 [acesso em 2010 Apr 8]. Disponível em: http://www.ifi.uzh.ch/groups/ ailab/people/nitschke/refs/Complexity-Economy.pdf.

35. Ruhl JB. The Fitness of Law: Using Complexity Theory to Describe the Evolution of Law and Society and Its Practical Meaning for Democracy, 
49 Vand. L. Rev. 1407 (1996). [acesso em 2009 Apr 8]. Disponível em: http://papers.ssrn.com/sol3/papers.cfm?abstract_id=1353428.

36. Schwartz G, Trindade AFR, Gloeckner AMEJ. Autopoiese e constituição. Os limites da hierarquia e as possibilidades da circularidade. Passo Fundo, RS: UPF; 2005.

37. Kurakin A. Order without design. Theoretical Biology and Medical Modelling. 2010; 7: 12. [acesso em 2009 Apr 8]. Disponível em: http://www.tbiomed.com/content/pdf/1742-4682-7-12.pdf.

38. Almeida-Filho N, Coutinho D. Causalidade, contingência, complexidade: o futuro do conceito de risco. Physis [online]. 2007; 17(1). [acesso em 2010 Maio 7]. pp. 95-137. Disponível em: http://www.scielo.br/pdf/physis/v17n1/v17n1a07.pdf
39. Jayasinghe S. Conceptualising population health: from mechanistic thinking to complexity science. Emerg Themes Epidemiol. 2011; 8: 2. Disponível em: http://www.ete-online.com/content/8/1/2

40. Nobrega M. O subdesenvolvimento não se improvisa. Veja 2009; 2129 / 9 de setembro de 2009. Disponível em: http://veja.abril.com.br/090909/ subdesenvolvimento-nao-improvisa-p-133.shtml

41. Seabra O, Carvalho M, Leite JC. Território e Sociedade. Entrevista com Milton Santos. São Paulo: Fundação Perseu Abramo; 2006.

42. Brasil. Ministério da Saúde. Secretaria de Atenção à Saúde. Política Nacional de Atenção Básica. $4^{a}$ ed. Brasília: Ministério da Saúde; 2007.

43. ABES. Dia Mundial da Saúde. Mesa redonda discute a saúde da população. Jornal da ABES. 2010; 1(7). Porto Alegre. Disponível em: http://www.abes-rs.org.br/jornal/jornal_da_abes-rs_a1n7_red.pdf 